This item was submitted to Loughborough's Research Repository by the author.

Items in Figshare are protected by copyright, with all rights reserved, unless otherwise indicated.

\title{
Sloshing frequencies of longitudinal modes for a liquid contained in a trough
}

PLEASE CITE THE PUBLISHED VERSION

PUBLISHER

(c) Cambridge University Press

VERSION

VoR (Version of Record)

LICENCE

CC BY-NC-ND 4.0

REPOSITORY RECORD

Mclver, P., and M. Mclver. 2019. "Sloshing Frequencies of Longitudinal Modes for a Liquid Contained in a Trough". figshare. https://hdl.handle.net/2134/4289. 
This item was submitted to Loughborough's Institutional Repository (https://dspace.lboro.ac.uk/) by the author and is made available under the following Creative Commons Licence conditions.

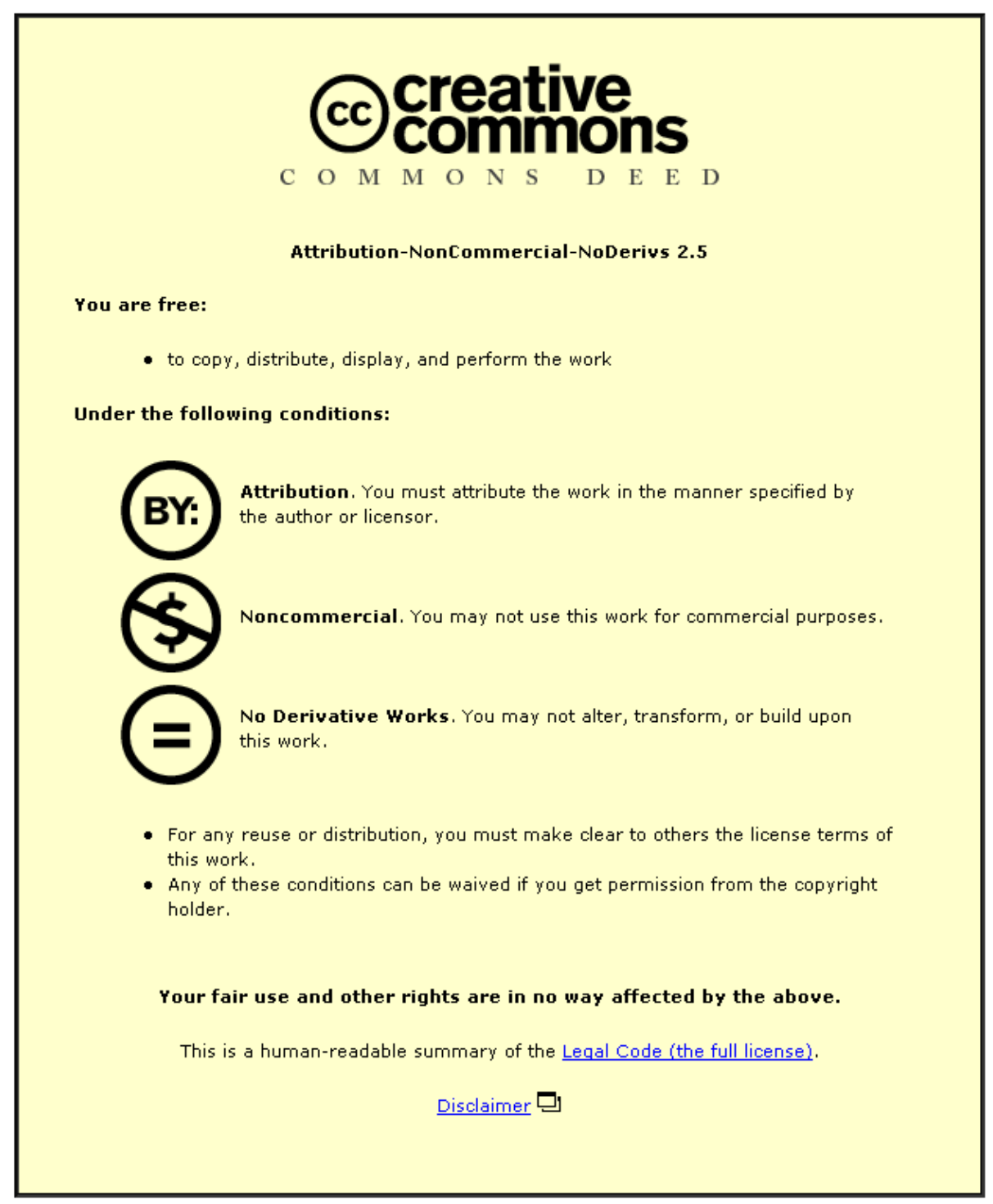

For the full text of this licence, please go to: http://creativecommons.org/licenses/by-nc-nd/2.5/ 


\title{
Sloshing frequencies of longitudinal modes for a liquid contained in a trough
}

\author{
By P. MCIVER AND M. MCIVER \\ Department of Mathematical Sciences, Loughborough University of Technology, Loughborough, \\ Leicestershire, LE11 3TU, UK
}

(Received 16 September 1992)

The sloshing under gravity is considered for a liquid contained in a horizontal cylinder of uniform cross-section and symmetric about a vertical plane parallel to its generators. Much of the published work on this problem has been concerned with twodimensional, transverse oscillations of the fluid. Here, attention is paid to longitudinal modes with variation of the fluid motion along the cylinder. There are two known exact solutions for all modes; these are for cylinders whose cross-sections are either rectangular or triangular with a vertex semi-angle of $\frac{1}{4} \pi$. Numerical solutions are possible for an arbitrary geometry but few calculations are reported in the open literature. In the present work, some general aspects of the solutions for arbitrary geometries are investigated including the behaviour at low and high frequency of longitudinal modes. Further, simple methods are described for obtaining upper and lower bounds to the frequencies of both the lowest symmetric and lowest antisymmetric modes. Comparisons are made with numerical calculations from a boundary element method.

\section{Introduction}

A liquid with a free surface within a bounded container has certain natural frequencies of oscillation such that excitation of the container at a nearby frequency will lead to large-amplitude fluid motions. There are many circumstances where it is important to know these natural frequencies; for example, during the transportation of liquid cargoes a substantial oscillation of the fluid may lead to a loss of stability of the carrying vehicle.

If only the natural frequencies are needed, that is the details of the fluid motion are not required, then it is sufficient to use the linearized equations for the irrotational motion of an inviscid fluid. Choose Cartesian coordinates $(x, y, z)$ with origin in the mean free surface and $y$ directed vertically downwards. Let $\bar{C}$ denote the wetted surface of the container and $\vec{F}$ the free surface of the liquid. Removing a time-harmonic factor, the free oscillations of the fluid may be described by a velocity potential $\Phi(x, y, z)$ satisfying Laplace's equation

$$
\Phi_{x x}+\Phi_{y y}+\Phi_{z z}=0
$$

within the fluid, the condition of no flow through the solid surface

$$
\Phi_{n}=0 \text { on } \bar{C},
$$

where $n$ is an outward normal coordinate, and the free-surface condition

$$
K \Phi+\Phi_{y}=0 \text { on } \bar{F},
$$

where $K=\omega^{2} / g, \omega$ is the radian frequency of the oscillations and $g$ is the acceleration due to gravity. This is a homogeneous problem and solutions will exist only for 


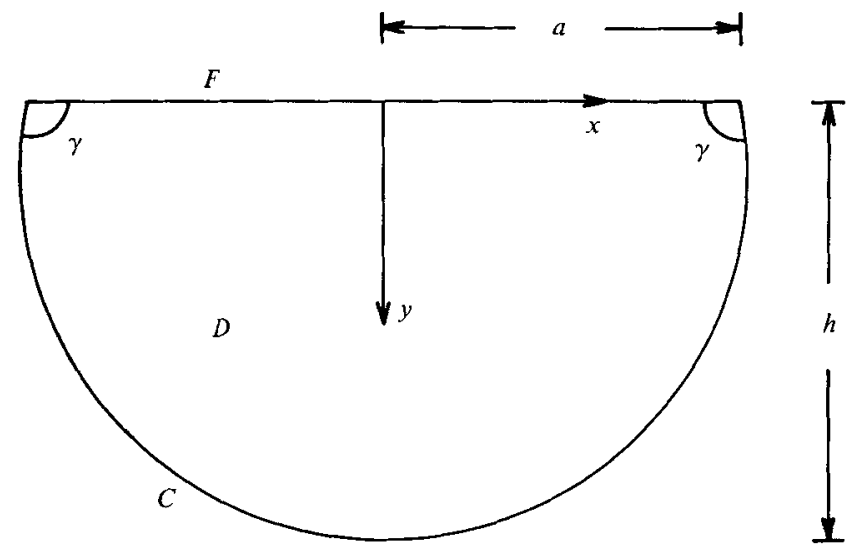

FigurE 1. Definition sketch.

particular values of $K$ corresponding to the natural frequencies. In other words (1.1)-(1.3) constitute an eigenvalue problem in which the eigenvalue $K$ appears in the free-surface boundary condition (1.3).

There is now a substantial body of work on the solution of (1.1)-(1.3) for the eigenvalue $K$ and extensive references are given by Moiseev \& Petrov (1966) and Fox \& Kuttler (1983). Most of this work is concerned with either two-dimensional oscillations or with motions in containers having a vertical axis of symmetry. A third, and important, class of problems is that of non-transverse oscillations in horizontal cylinders, or troughs, of arbitrary cross-section and these geometries are the subject of the present paper. Let such a container have parallel, horizontal generators with vertical endwalls, located at $z=0$ and $z=l$, that are perpendicular to the cylinder generators. The condition of no flow through the endwalls may be satisfied by taking

provided that

$$
\begin{gathered}
\Phi(x, y, z)=\phi(x, y) \cos p z \\
p=m \pi / l,
\end{gathered}
$$

where $m$ is any non-negative integer. (The two-dimensional problem of transverse oscillations corresponds to the case $m=0$.) The eigenvalue problem (1.1)-(1.3) now reduces to the field equation

$$
\left(\nabla^{2}-p^{2}\right) \phi=0 \text { in } D,
$$

where $D$ is the fluid domain and $\nabla^{2}$ is the two-dimensional Laplacian, together with the boundary conditions

and

$$
\begin{gathered}
\phi_{n}=0 \text { on } C \\
K \phi+\phi_{y}=0 \text { on } F .
\end{gathered}
$$

Here $C$ and $F$ denote respectively the cross-sections of the wetted container and free surface lying in the $(x, y)$-plane. In particular, $F$ will be taken to be the interval $|x| \leqslant a$ in $y=0$ so that the free surface is of width $2 a$. The geometry and coordinate system for the cross-sectional oscillation are illustrated in figure 1 . In the present work attention will be restricted to containers that are symmetric about $x=0$ so that all modes of oscillation are either symmetric or antisymmetric in $x$.

There are a small number of exact solutions to (1.6)-(1.8). If the container is rectangular and of depth $h$ then separation of variables yields the solution

$$
\phi=\cos \alpha(x-a) \cosh k(y-h),
$$




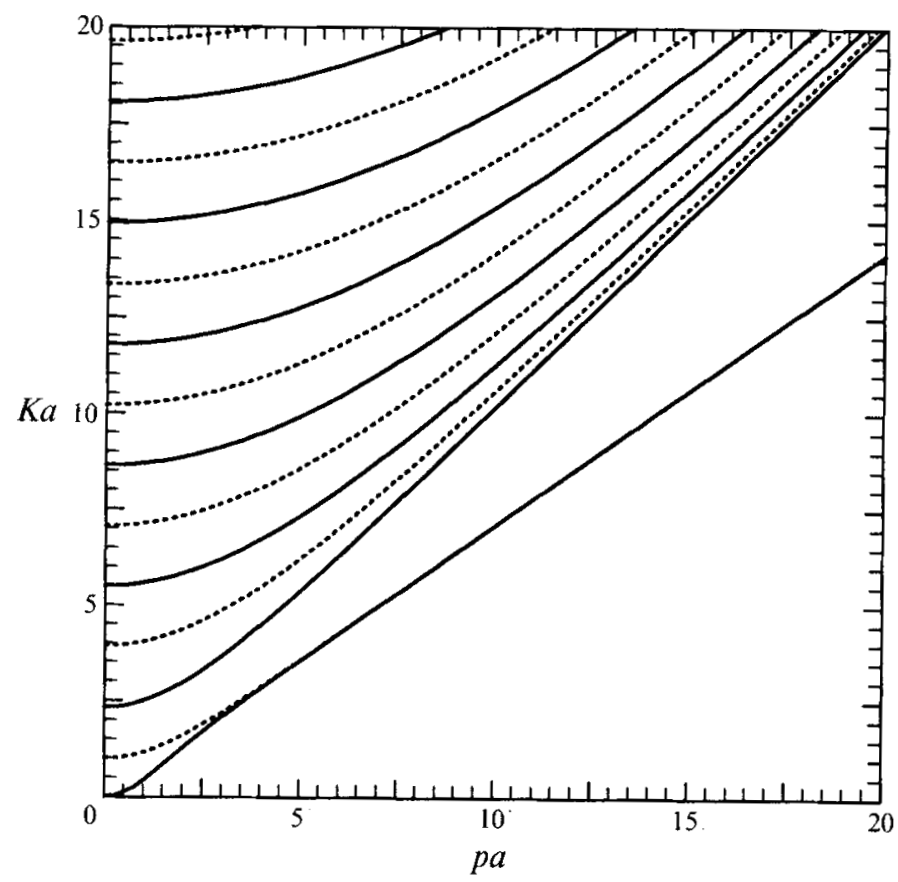

FIGURE 2. Symmetric modes (- - ) and antisymmetric modes (----) for a triangular basin with vertex semi-angle $\frac{1}{4} \pi$.

where

$$
\alpha=M \pi / 2 a, \quad k=\left(\alpha^{2}+p^{2}\right)^{\frac{1}{2}} ; \quad M=0,1,2, \ldots .
$$

The corresponding values of the frequency parameter are given by

$$
K=k \tanh k h \text {. }
$$

For any given geometry there is a doubly infinite set of modes identified by the integers $m$ and $M$ in the definitions of $p$ and $\alpha$. For even $M$ the fluid motion is symmetric about $x=0$ while for odd $M$ the motion is antisymmetric. This interleaving of infinite sets of symmetric and antisymmetric modes is typical of all geometries symmetric about $x=0$. The only other geometry for which there is an exact solution for all modes is a triangular basin whose sides are inclined at an angle $\frac{1}{4} \pi$ to the horizontal; this solution is described by Lamb (1932, $\$ 258$ and 261) and the relationship between the eigenvalue $K$ and $p$ is plotted in figure 2. Each curve of $K a$ against $p a$ gives the eigenfrequencies of a particular mode of oscillation. Note that for any particular mode the limits of $K a$ and $p a$ tending to infinity are equivalent and in the following this will be referred to as the high-frequency limit. For a given container length $l$, possible values of $p$ are given by (1.5) and the eigenvalues can then be read off from the figure. For a fixed $p$, modes are alternately symmetric and antisymmetric with increasing frequency. Of particular practical importance are the lowest symmetric mode, which may be excited by longitudinal motion of the container parallel to the cylinder generators, and the lowest antisymmetric mode, which may be excited by horizontal forcing perpendicular to the cylinder generators. In addition to the above-mentioned exact solutions for all modes, the frequencies of the symmetric modes of oscillation for a triangular basin with sides inclined at $\frac{1}{6} \pi$ to the horizontal have been obtained by Packham (1980).

Numerical solutions of (1.6)-(1.8) for a circular cross-section filled to arbitrary depth were found by Moiseev \& Petrov (1966) using the Ritz variational method but only a 
few results were presented. More recently, McIver \& Smith (1987) have given an approximate method, based on the mild-slope equation, that is applicable to nonbulbous containers of arbitrary cross-section $C$. Evans \& Linton (1993) have given an accurate series method of solution for a half-full horizontal circular cylinder. Numerical solutions using, for example, the Ritz method or the boundary element method are possible for an arbitrary contour $C$; the latter method is used here for purposes of illustration. Experimental measurements of the natural frequencies have been made for longitudinal sloshing in a trough of circular cross-section by a number of workers, see for example the recent paper by Kobayashi et al. (1989).

A number of authors have considered high-frequency approximations to sloshing frequencies and a recent discussion is given by Davis (1992). In particular, Fox \& Kuttler (1983) have conjectured that in the two-dimensional case $(p=0)$

$$
K a \sim \frac{1}{2} \pi\left(M+\frac{1}{2}-\frac{\pi}{4 \gamma}\right)
$$

as the mode number $M$ tends to infinity, showing how the eigenvalues depend on the angle of intersection $\gamma$ between the container and the free surface. For sufficiently large $K a$ the motion is essentially confined to a thin surface layer so it is not surprising that there can be such a dependence on $\gamma$. The case of non-zero $p$ is considered in the present work. For $0<\gamma<\frac{1}{2} \pi$ the high-frequency behaviour of some modes is found to be directly related to the edge-wave solutions for a beach of the same angle; Lamb (1932, p. 448) remarks that Stokes' edge-wave solution is recovered at high frequency for the special case of the lowest two modes in a triangular basin with intersection angle $\frac{1}{4} \pi$ and this may be observed in figure 2. The lowest two modes asymptote to $K=p \sin \gamma$, $\gamma=\frac{1}{4} \pi$, which is the edge-wave dispersion relation, while higher modes asymptote to $K=p$. For $\frac{1}{2} \pi \leqslant \gamma \leqslant \pi$, edge-wave solutions do not exist and the high-frequency asymptote is $K=p$ for all modes.

Many useful results in sloshing problems follow from the max-min definition of eigenvalues as given in Chapter 6 of Courant \& Hilbert (1953). For example, simple bounds on the eigenfrequencies for a contour $C$ with free surface $F$ may be obtained by comparing with known solutions for other containers. Thus, for fixed $p$, the eigenfrequencies of each mode are bounded above by the solution for a contour entirely containing $C$ and below by the solution for a contour entirely contained within $C$, provided each contour has the same free surface $F$. The definition of eigenvalues in terms of an extremum principle is also the basis of the Ritz method used by Moiseev \& Petrov (1966) and others.

The problem of liquid sloshing in a trough is closely related to the propagation of waves in a channel of constant cross-section. If the cosine factor in (1.4) is replaced by $\exp (\mathrm{i} z z)$ then (1.6) is unaltered but the potential then describes waves propagating in the $z$-direction with wavenumber $p$. With this interpretation, frequency curves such as those in figure 2 give the dispersion relation for such waves. This interpretation has been made by Shi \& Yih (1984) who make detailed calculations for a channel of trapezoidal cross-section. Unfortunately, their results appear to be in error as many do not satisfy elementary bounds derived in the way indicated in the previous paragraph.

In the present work some of the more general aspects of the solutions of (1.6)-(1.8) are examined for an arbitrary symmetric contour $C$, with particular reference to high and low frequencies. In $\S 2$, an approximate solution is given for a container whose bed is horizontal and walls are close to the vertical. This solution is used to illustrate how the container geometry determines the low- and high-frequency behaviour of the lowest symmetric mode and the high-frequency behaviour of the lowest antisymmetric 
mode. The methods described in this paper are unable to determine the low-frequency asymptotic behaviour of the lowest antisymmetric mode. The high-frequency results are extended in $\S 3$ to containers of arbitrary geometry. In $\$ 4$ and 5 simple methods are given by estimating the eigenvalues through the use of upper and lower bounds. The upper bounds are obtained by a simple application of the Ritz method while the lower bounds follow from results on maximum principles that do not appear to have been used previously in sloshing problems. In each case the choice of trial function is guided by the high-frequency approximations to the eigenfunctions discussed in $\S 3$. Finally results are presented and discussed in $\S 6$.

\section{Containers with almost-vertical walls}

A number of the features of the solutions of (1.6)-(1.8), for containers of arbitrary shape, may be illustrated by considering symmetric containers with a horizontal bottom and sides which differ little from the vertical. The method of solution follows Evans \& Mclver (1991) who considered wave trapping by almost-vertical walls. The horizontal bottom is at $y=h$ and the walls of the container are at $x= \pm(a-\epsilon f(y))$, where the non-dimensional parameter $\epsilon$ is taken to be small. It will be assumed, without loss of generality, that $f(0)=0$. All modes are either symmetric or antisymmetric about $x=0$ so that only $0 \leqslant x \leqslant a-\epsilon f(y)$ need be considered provided an appropriate condition is imposed at $x=0$. The boundary conditions to be satisfied on the container contour $C$ are therefore

$$
\begin{array}{lrl} 
& \phi_{y}=0 \text { on } y=h \\
\text { and } & \phi_{n}=0 \text { on } x=a-\epsilon f(y), \\
\text { with } & \phi_{x}=0 \text { on } x=0 \\
\text { for symmetric modes and } & \phi=0 \text { on } x=0
\end{array}
$$

for antisymmetric modes. The field equation (1.6) and the free-surface condition (1.8) are unchanged. The sidewall condition (2.2) may be transferred to $x=a$ by Taylor series expansion to get

$$
\phi_{x}=\epsilon\left(f(y) \phi_{x x}-f^{\prime}(y) \phi_{y}\right)+O\left(\epsilon^{2}\right) \quad \text { on } \quad x=a .
$$

A solution is now sought in the rectangular region $0 \leqslant x \leqslant a, 0 \leqslant y \leqslant h$. From wavemaker theory the solution for symmetric modes, satisfying all of the conditions of the problem apart from the wall condition (2.5), takes the form

$$
\phi=\cos \alpha x \psi_{0}(y)+\chi(x, y),
$$

where

$$
\chi(x, y)=\sum_{n=1}^{\infty} A_{n} \cosh \alpha_{n} x \psi_{n}(y)
$$

and

$$
\psi_{n}(y)=N_{n}^{-\frac{1}{2}} \cos k_{n}(y-h), \quad n=0,1,2, \ldots,
$$

with

$$
N_{n}=\frac{1}{2}\left(h+\sin 2 k_{n} h / 2 k_{n}\right) \text {. }
$$

(The coefficient of the first term on the right-hand side of (2.6) has been chosen as unity without loss of generality.) Here

and

$$
\begin{aligned}
& \alpha_{n}=\left(k_{n}^{2}+p^{2}\right)^{\frac{1}{2}} \\
& \alpha=\left(k^{2}-p^{2}\right)^{\frac{1}{2}},
\end{aligned}
$$

where the $k_{n}$ are the real, positive roots of

$$
K+k_{n} \tan k_{n} h=0, \quad n=1,2,3, \ldots,
$$


$k$ is the real, positive root of (1.11) and $k_{0}=-\mathrm{i} k$. The functions $\psi_{n}(y), n=0,1,2, \ldots$ form a complete orthonormal set over $[0, h]$ so that from the definition of $\chi(x, y)$ in $(2.7)$

$$
\int_{0}^{h} x(x, y) \psi_{0}(y) \mathrm{d} y=0, \quad 0 \leqslant x \leqslant a .
$$

As $\epsilon \rightarrow 0$, so that the walls are vertical, the solution to within a constant factor is given by (1.9)-(1.11) with $M$ an even integer or zero. Comparison of (1.9) and (2.6) suggests that

$$
\chi(x, y) \rightarrow 0 \text { as } \epsilon \rightarrow 0 .
$$

The substitution of (2.6) into the wall boundary condition (2.5) gives

$$
-\alpha \sin \alpha a \psi_{0}(y)+\chi_{x}(a, y)=\epsilon\left\{f(y)\left(-\alpha^{2} \cos \alpha a\right) \psi_{0}(y)-f^{\prime}(y) \cos \alpha a \psi_{0}^{\prime}(y)\right\}+\ldots,
$$

where terms involving derivatives of $\chi$ have been dropped from the right-hand side because of (2.14). Multiplication throughout by $\psi_{0}(y)$, integration over the depth, and the use of (2.13) yields

$$
\alpha \tan \alpha a=\epsilon\left\{\alpha^{2} \int_{0}^{h} f(y) \psi_{0}^{2}(y) \mathrm{d} y+\int_{0}^{h} f^{\prime}(y) \psi_{0}^{\prime}(y) \psi_{0}(y) \mathrm{d} y\right\}+\ldots
$$

As an example of the use of (2.16), a balance of terms is possible if $\alpha a=O\left(\epsilon^{\frac{1}{2}}\right)$ in which case (2.16) reduces to

$$
\alpha^{2} a=\epsilon \int_{0}^{h} f^{\prime}(y) \psi_{0}^{\prime}(y) \psi_{0}(y) \mathrm{d} y=\frac{-2 k^{2} \epsilon}{2 k h+\sinh 2 k h} \int_{0}^{h} f^{\prime}(y) \sinh 2 k(h-y) \mathrm{d} y .
$$

In the solution for a rectangular container, equations (1.9)-(1.11), the lowest symmetric mode has $\alpha=0$ and (2.17) describes a small perturbation from this situation. Clearly, $\alpha$ may be either real or imaginary. For $k h \ll 1$, (2.17) yields the lowfrequency approximation

Now from (2.11)

$$
\alpha^{2} a=-\frac{k^{2} \epsilon}{h} \int_{0}^{h} f(y) \mathrm{d} y .
$$

$$
p^{2}=k^{2}-\alpha^{2}=k^{2}\left(1+\frac{\epsilon}{a h} \int_{0}^{h} f(y) \mathrm{d} y\right) \sim \frac{2 k^{2} a h}{A},
$$

where

$$
A=2\left(a h-\epsilon \int_{0}^{h} f(y) \mathrm{d} y\right)
$$

is the cross-sectional area of the container. Finally using the low-frequency approximation to (1.11), $K \sim k^{2} h,(2.19)$ gives

$$
K \sim \frac{A}{2 a} p^{2}
$$

as the low-frequency behaviour of the lowest symmetric mode. This result may be shown to be true for arbitrary containers as follows. Apply Green's theorem to $\phi$, satisfying (1.6)-(1.8), and $\psi=1$ over the fluid domain $D$ to obtain

$$
K=\frac{\iint_{A} \phi \mathrm{d} A}{\int_{F} \phi \mathrm{d} x} p^{2} .
$$


As $K \rightarrow 0$ the potential $\phi$ tends to a constant over $D$ and (2.22) reduces to (2.21).

For high frequencies, as $k h \rightarrow \infty$, Watson's lemma gives

$$
\int_{0}^{h} f(y) \psi_{0}^{2}(y) \mathrm{d} y \sim \frac{f^{\prime}(0)}{2 k}
$$

and

$$
\int_{0}^{h} f^{\prime}(y) \psi_{0}^{\prime}(y) \psi_{0}(y) \mathrm{d} y \sim-k f^{\prime}(0),
$$

and (2.16) becomes for $k \gg \alpha$ (that is $p$ not close to zero)

$$
\alpha a \tan \alpha a=-\epsilon k a f^{\prime}(0)=-k a \cot \gamma,
$$

where $\gamma$ is the angle between the sidewall and the free surface. For $0<\gamma<\frac{1}{2} \pi$, the right-hand side of (2.25) is negative and there is an infinite set of positive real roots for $\alpha a$. For these roots

$$
\alpha a \sim\left(n+\frac{1}{2}\right) \pi, \quad n=0,1,2, \ldots, \quad \text { as } k a \rightarrow \infty
$$

for fixed mode number $n$ and from (2.11)

$$
p \sim k \text { as } k a \rightarrow \infty \text {. }
$$

There is also an imaginary root given by $\alpha=\mathrm{i} \beta$, say, where $\beta$ is the positive real root of

and

$$
\beta a \tanh \beta a=k a \cot \gamma
$$

so that

$$
\begin{aligned}
& \beta a \sim k a \cot \gamma \text { as } k a \rightarrow \infty \\
& p \sim k \operatorname{cosec} \gamma \text { as } k a \rightarrow \infty \text {. }
\end{aligned}
$$

For $\frac{1}{2} \pi<\gamma<\pi$, the right-hand side of (2.25) is positive and there are only real roots so that the asymptotic behaviour is given by (2.26)-(2.27).

A similar analysis may be performed for antisymmetric modes by writing the potential as

$$
\phi=\sin \alpha x \psi_{0}(y)+\chi(x, y),
$$

and the equivalent of $(2.25)$ is

$$
\alpha a \cot \alpha a=k a \cot \gamma
$$

As $k a \rightarrow \infty$, the roots of (2.32) behave in the same way as those of (2.25) with the exception that

$$
\alpha a \sim n \pi, \quad n=1,2,3, \ldots, \quad \text { as } k a \rightarrow \infty
$$

for the positive real roots.

\section{High-frequency behaviour for an arbitrary container}

By using the results derived in the previous section as a guide, high-frequency approximations to the eigenvalues and eigenfunctions are now given for symmetric containers of arbitrary geometry. The reader is reminded that, for any particular mode, the limits of $K a$ and $p a$ tending to infinity are equivalent and are together referred to as the high-frequency limit. The arguments used in this section are informal but all of the results described have been verified for a number of geometries by examining numerical solutions. It is possible that formal proofs of the results can be obtained by methods similar to those used by Davis (1965) for the two-dimensional problem.

In a surface layer away from the sidewalls the solution for symmetric modes will be of the form

$$
\phi=\cos \alpha x \mathrm{e}^{-K y},
$$


where $K=\omega^{2} / g$ and

$$
\alpha=\left(K^{2}-p^{2}\right)^{\frac{1}{2}}
$$

For most modes $\alpha$ is real but, as seen in $\S 2$, there is the possibility of a finite number of modes for which $\alpha$ is pure imaginary. For real $\alpha$, rewrite (3.1) as

$$
\phi=\frac{1}{2}\left(\mathrm{e}^{\mathrm{i} \alpha(x+a)} \mathrm{e}^{-2 \mathrm{i} \alpha a}+\mathrm{e}^{-\mathrm{i} \alpha(x+a)}\right) \mathrm{e}^{\mathrm{i} \alpha a} \mathrm{e}^{-K y} .
$$

In this high-frequency approximation, the sides of the container will appear as plane walls inclined at an angle $\gamma$ to the horizontal free surface. Thus, a solution is possible if (3.3) describes the reflection of a plane wave from a beach of slope $\tan \gamma$ at $x=-a$ (by symmetry it is necessary to consider only one of the intersection points). The eigenvalue relation is therefore

where

$$
\begin{gathered}
\mathrm{e}^{-2 \mathrm{i} \alpha a}=R, \\
R=\mathrm{e}^{\mathrm{i} \delta}
\end{gathered}
$$

is the coefficient of reflections for such a beach and $\delta$, which is a function of $\alpha$ and $p$, may be calculated from the work of Peters (1952). Thus for symmetric modes

$$
\alpha a \sim n \pi-\frac{1}{2} \delta,
$$

where $n$ is an integer, and from (3.2)

as $K \rightarrow \infty$ for fixed $n$.

$$
p \sim K
$$

For imaginary $\alpha$ write $\alpha=\mathrm{i} \beta$ so that

$$
\phi=\cosh \beta x \mathrm{e}^{-K y} \sim \frac{1}{2} \mathrm{e}^{\beta a} \mathrm{e}^{-\beta(x+a)} \mathrm{e}^{-K y}
$$

as the rim at $x=-a$ is approached. A solution to the sloshing problem is possible provided the asymptotic form in (3.8) may be matched to a solution that decays exponentially way from the plane wall intersecting the surface at $x=-a$. Solutions of the latter type are the edge waves given by Ursell (1952) for which the potential

$$
\phi_{\mathrm{e}} \sim B \mathrm{e}^{-(x+a) p \cos (2 m+1) \gamma} \mathrm{e}^{-y p \sin (2 m+1) \gamma}, \quad m=0,1,2, \ldots, \quad \text { as } \quad x+a \rightarrow \infty,
$$

where the number of modes is equal to the greatest integer contained in $\frac{1}{2}+\pi / 4 \gamma$ and $B$ is an amplitude. Thus (3.8) yields a high-frequency approximation for the sloshing problem provided

$$
\beta \sim p \cos (2 m+1) \gamma, \quad K \sim p \sin (2 m+1) \gamma .
$$

There are no edge-wave modes, and hence no sloshing modes of this type for $\gamma \geqslant \frac{1}{2} \pi$, while for $0<\gamma<\frac{1}{2} \pi$ the number of edge-wave modes increases indefinitely as $\gamma$ approaches zero.

Approximations to the antisymmetric modes are constructed by taking

$$
\phi=\sin \alpha x \mathrm{e}^{-k y}
$$

and following the above procedure. When $\alpha$ is real the eigenvalue relation is

so that

$$
\mathrm{e}^{-2 \mathrm{i} \alpha a}=-R
$$

The relations for imaginary $\alpha$ are similar to the symmetric mode case, that is

$$
\phi=\sinh \beta x \mathrm{e}^{-k y},
$$

with $\beta$ and $K$ given by (3.10). Thus, there are pairs of modes, one symmetric and one antisymmetric, for which the asymptotic behaviour is given by (3.10) for a valid choice of $m$. For $\gamma=\frac{1}{4} \pi$ then there is only a single edge-wave mode so that for the triangular- 
basin solution in figure 2 only the lowest symmetric and lowest antisymmetric mode have an edge-wave character at high frequency. A triangular basin with $\gamma=\frac{1}{12} \pi$, for example, will have three pairs of modes whose high-frequency behaviour is related to edge-wave solutions.

High-frequency approximations of the above type will be valid for small $p$ provided $K$ is large, that is the mode number is high. For $p=0$, Isaacson (1950) gives

$$
\delta=\frac{\pi}{2}\left(\frac{\pi}{2 \gamma}-1\right)
$$

(correcting a typographical error) and using this in (3.6) and (3.13) recovers the highmode approximation (1.12).

\section{Upper bounds for the lowest symmetric and antisymmetric modes}

The Ritz variational procedure has been used extensively in sloshing problems (e.g. Moiseev \& Petrov 1966) to furnish approximations to the eigenfrequencies. In particular, for a fixed $p$, the method gives upper bounds on the values of $K$ for all modes. Applying the results described by Courant \& Hilbert (1953, Chapter 6) gives for the lowest mode

$$
p^{2} \geqslant \frac{K \int_{F} w^{2} \mathrm{~d} x-\iint_{A}|\nabla w|^{2} \mathrm{~d} x \mathrm{~d} y}{\iint_{A} w^{2} \mathrm{~d} x \mathrm{~d} y} \equiv \mathscr{U}(K ; w),
$$

where $A$ denotes the fluid in the container cross-section and $w$ is a suitable trial function. Evaluation of $p_{1}^{2}=\mathscr{U}(K ; w)$ for given $K$ and $w$ yields a lower bound for $p$, that is $p \geqslant p_{1}$. It is more natural to specify $p$, which depends on the container length from (1.5), and seek the corresponding $K$; this may be achieved by solving

$$
p^{2}=\mathscr{U}\left(K_{\mathrm{u}} ; w\right)
$$

for $K_{\mathrm{u}}$ numerically, then $K \leqslant K_{\mathrm{u}}$. For fixed $p$, the inequality (4.1) may be used to furnish bounds on $K$ for any mode by choosing trial functions that are orthogonal to the eigenfunctions of all modes of lower frequency. Here only the lowest two mode are considered. The lowest mode is symmetric, that is an even function of $x$, so a bound for the second lowest, antisymmetric, mode is obtained by choosing a trial function that is an odd function of $x$.

The container contour $C$ is specified by

$$
x= \pm a g(y / a), \quad 0 \leqslant y \leqslant h,
$$

where $h$ is the container depth and $g$ is a single-valued function (restricting $g$ in this way covers most containers of practical interest) satisfying

$$
g(0)=1 \quad \text { and } \quad g^{\prime}(0)=-\cot \gamma
$$

Consider first containers for which $\frac{1}{2} \pi \leqslant \gamma<\pi$. In order to obtain good bounds for $K$, the trial function $w$ should be 'close' to the exact eigenfunction for the mode under consideration. For the lowest symmetric mode a suitable choice is

so that

$$
w=\cos \alpha x \cosh k(y-h),
$$

$$
\mathscr{U}(K ; w)=\frac{4 K a \cosh ^{2} k h J_{5}-\alpha^{2} a^{2}\left(J_{1}-J_{2}+J_{3}-J_{4}\right)+k^{2} a^{2}\left(J_{1}+J_{2}-J_{3}-J_{4}\right)}{a^{2}\left(J_{1}+J_{2}+J_{3}+J_{4}\right)},
$$


and for the lowest antisymmetric mode a suitable choice is

so that

$$
w=\sin \alpha x \cosh k(y-h),
$$

$$
\mathscr{U}(K ; w)=\frac{4 K a \cosh ^{2} k h J_{6}+\alpha^{2} a^{2}\left(J_{1}+J_{2}+J_{3}+J_{4}\right)-k^{2} a^{2}\left(J_{1}-J_{2}-J_{3}+J_{4}\right)}{a^{2}\left(-J_{1}+J_{2}-J_{3}+J_{4}\right)} .
$$

Here $k$ is the solution of (1.11) and

$$
\begin{gathered}
J_{1}=2 \int_{0}^{h / a} g(Y) \mathrm{d} Y=\frac{A}{a^{2}}, \quad J_{2}=\frac{1}{\alpha a} \int_{0}^{h / a} \sin [2 \alpha a g(Y)] \mathrm{d} Y, \\
J_{3}=2 \int_{0}^{h / a} g(Y) \cosh [2 k a(Y-h / a)] \mathrm{d} Y \\
J_{4}=\frac{1}{\alpha a} \int_{0}^{h / a} \sin [2 \alpha a g(Y)] \cosh [2 k a(Y-h / a)] \mathrm{d} Y \\
J_{5}=\frac{\sin 2 \alpha a}{2 \alpha a}+1, \quad J_{6}=\frac{\sin 2 \alpha a}{2 \alpha a}-1
\end{gathered}
$$

In the above $A$ is the cross-sectional area of the container. For an arbitrary shape function $g(Y)$ the integrals in (4.9) must be evaluated numerically.

It remains to choose suitable values for $\alpha$. The high-frequency analysis of $\S 3$ suggests that the values given by (3.6) and (3.13), with $n=0$, are appropriate choices for the lowest symmetric and lowest antisymmetric mode respectively. Unfortunately the expression for the phase $\delta$ of the reflection coefficient, which may be found from the results of Peters (1952), depends on $\alpha$ and $p$ in an extremely complicated way. However, the value for $p=0$ is given by (3.15) and this was used for all $p$ with good effect when $\frac{1}{2} \pi \leqslant \gamma<\pi$ even at low frequency. Other possible choices for $\alpha$ are integer multiples of $\frac{1}{2} \pi$ as suggested by the high-frequency behaviour in (2.26) and (2.33) but these were less effective. For $0<\gamma<\frac{1}{2} \pi, \alpha$ is replaced by $\mathrm{i} \beta$ throughout with $\beta=K \cot \gamma$ as suggested by the high-frequency behaviour described in $\S 3$. Choosing $\beta=k \cot \gamma$, where $k$ is the root of (1.11) gave marginally poorer results in general.

In the low-frequency limit for the lowest symmetric mode, that is as $K a \rightarrow 0$, any choice such that $\alpha a$ also approaches zero at the same rate or faster gives

$$
\mathscr{U} \sim 2 \mathrm{Ka} / \mathrm{A},
$$

reproducing the known behaviour of the exact solution given by (2.21). For a fixed $\alpha$, used when $\frac{1}{2} \pi \leqslant \gamma<\pi$, the high-frequency behaviour of $\mathscr{U}$ is dominated by $J_{i}, i=3,4$, 5,6. By Watson's lemma

$$
J_{3} \sim \frac{\mathrm{e}^{2 k h}}{2 k a} \text { and } \quad J_{4} \sim \frac{\mathrm{e}^{2 k h}}{2 k a} \frac{\sin 2 \alpha a}{2 \alpha a}
$$

so that

$$
\mathscr{U} \sim K^{2} \text { as } K \rightarrow \infty
$$

for both the symmetric and antisymmetric modes. For $\alpha=\mathrm{i} \beta=\mathrm{i} K \cot \gamma$, used when $0<\gamma<\frac{1}{2} \pi, \mathscr{U}$ is dominated by $J_{i}, i=4,5,6$. By Laplace's method

$$
J_{4} \sim \frac{\mathrm{e}^{2(k h+k a \cot \gamma)}}{8 k^{2} a^{2}} \sin ^{2} \gamma \tan \gamma,
$$


assuming $g(Y)$ has its maximum at $Y=0$, so that in this case

$$
\mathscr{U} \sim K^{2} \operatorname{cosec}^{2} \gamma \text { as } K \rightarrow \infty
$$

for all modes. Combining (4.12) and (4.14) with (4.1) recovers the high-frequency behaviour of the exact solution given in (3.7) and (3.10). In other words the simple choices of trial function given here are able to reproduce both the high- and lowsurface asymptotics of the lowest symmetric mode and the high-frequency asymptotics of the lowest antisymmetric mode.

\section{Lower bounds for the lowest symmetric and antisymmetric modes}

If the geometry of the container, (and therefore the values $p$ may take), is fixed, a lower bound for the eigenvalue $K$ may be obtained using a theorem due to Protter \& Weinberger (1984). To the authors' knowledge, this theorem has not previously been applied to sloshing problems and so for completeness, an appropriate version of the theorem is stated below and proved in the Appendix. A more general form of the theorem is stated and proved in Protter \& Weinberger (1984, p. 91).

THEOREM. Let $p$ and $K$ be given real values for which a non-zero solution of (1.6)-(1.8) exists. Let $w$ be positive on $D \cup C \cup F$ and satisfy

$$
\begin{gathered}
\nabla^{2} w-\beta^{2} w \leqslant 0 \text { in } D, \\
w_{n} \geqslant 0 \text { on } C,
\end{gathered}
$$

and

$$
K w+w_{y} \leqslant 0 \text { on } F
$$

for some non-negative number $\beta$, where $n$ denotes the outward normal direction from $D$, then

Proof. See Appendix.

$$
p \leqslant \beta \text {. }
$$

If a suitable function $w$ can be found the theorem provides an upper bound on $p$ for a given $K$. In practice, it is usually possible to choose $w$ such that $K$ is a monotonically increasing function of $\beta, \chi(\beta)$ say. In this case, it is more convenient to regard $p$ as fixed then $K_{1}=\chi(p)$ is a lower bound for $K=\chi(\beta)$ as $p \leqslant \beta$. Thus, when constructing particular forms for $w$, the parameter $\beta$ in (5.1) will be replaced by $p$ and the parameter $K$ in (5.3) will be replaced by $K_{1}$. Although $K_{1}$ is a lower bound for the value of $K$ associated with any of the modes, it will not give an accurate prediction of $K$ for all $p$, for any mode apart from the lowest symmetric one. However, it is possible to modify the theorem to provide an improved lower bound for the value of $K$ associated with the lowest antisymmetric mode which satisfies

$$
\phi=0 \text { on } x=0 \text {. }
$$

In this case, (5.4) still holds provided that a function $w$ may be found which is positive and satisfies (5.1)-(5.3) in that part of the region in which $x \geqslant 0$. The proof of this modified theorem is covered by the proof of the general theorem in Protter \& Weinberger (1984).

The accuracy of the bound depends on both the geometry and the choice of $w$. Before determining a suitable choice of $w$ for a general shaped container, it is instructive to consider a symmetric, triangular container filled to a depth $h$, with a free surface width equal to $2 a$ and angle of intersection between the container and the free surface equal to $\gamma$. A possible choice for $w$ is given by

$$
w=I_{0}(p r),
$$


where $I_{0}$ is a modified Bessel function and $r^{2}=x^{2}+(y-h)^{2}, r \geqslant 0$. Clearly $w>0$ everywhere and satisfies (5.1) and (5.2), putting $\beta=p$. On $y=0$,

$$
K_{1} w+w_{y}=K_{1} I_{0}\left[p\left(x^{2}+h^{2}\right)^{\frac{1}{2}}\right]-\frac{p h}{\left(x^{2}+h^{2}\right)^{\frac{1}{2}}} I_{1}\left[p\left(x^{2}+h^{2}\right)^{\frac{1}{2}}\right] .
$$

By using the recurrence relations for modified Bessel functions (Abramowitz \& Stegun 1965 ), it is possible to show that the right-hand side of this equation is a monotonically increasing function of $x^{2}$, provided that $K_{1}>0$. Thus, (5.3) is satisfied by putting $K=K_{1}$ and choosing $K_{1}$ so that the right-hand side of (5.7) equals zero at $x=a$, i.e.

$$
K_{1}=\frac{p \sin \gamma I_{1}(p a / \cos \gamma)}{I_{0}(p a / \cos \gamma)}
$$

using the equality $h=a \tan \gamma$. It is particularly interesting to determine the dependence of $K_{1}$ on $p$ at low and high frequencies. From (5.8) and the properties of modified Bessel functions,

$$
K_{1} \sim \frac{1}{2} p^{2} a \tan \gamma=\frac{A}{2 a} p^{2} \quad \text { as } \quad p a \rightarrow 0
$$

and

$$
K_{1} \sim p \sin \gamma \text { as } p a \rightarrow \infty,
$$

where $A$ is the cross-sectional area of the triangle. The low- and high-frequency behaviour of the lower bound is precisely the same as that obtained for the upper bound for the lowest symmetric mode in the previous section. Thus, (5.7) and (5.8) give the leading-order asymptotic behaviour of the value of $K$ associated with the lowest symmetric mode at low and high frequencies, confirming the predictions of (2.21) and (2.30).

The trial function $w$ given by (5.6) may be used to obtain a lower bound for $K$ for containers other than the triangular one. However, experience suggests that an improved numerical bound for the value of $K$ associated with the lowest symmetric mode is obtained using the function

$$
w=\cosh [p x \cos \sigma] \cosh [p(y-d) \sin \sigma],
$$

where $K_{1}$ is defined to be $\quad K_{1}=p \sin \sigma \tanh [p d \sin \sigma]$,

$\sigma \in\left[0, \frac{1}{2} \pi\right]$ and $p d \geqslant 0$. Clearly $w>0$ everywhere and satisfies (5.1) and (5.3), putting $\beta=p$ and $K=K_{1}$. The body boundary condition (5.2) is satisfied provided that $\sigma$ and $p d$ are chosen so that

$$
s(x, y)=n_{x} \cos \sigma \tanh [p x \cos \sigma]+n_{y} \sin \sigma \tanh [p(y-d) \sin \sigma] \geqslant 0 \text { on } C,
$$

where $n_{x}$ and $n_{y}$ are the $x$ - and $y$-components of the unit outward normal to $D$. If the body shape function $g(y / a)$ defined in (4.3) is restricted to the concave then (5.13) is automatically satisfied $\forall \sigma \in\left[0, \frac{1}{2} \pi\right]$ by choosing $p d=p y_{\mathrm{m}}$, where $y_{\mathrm{m}} / a$ is the value at which $g$ takes its maximum. This choice of $p d$ ensures that both terms in (5.13) are nonnegative but does not usually produce a value of $K_{1}$ which is a close lower bound for $K$. However, the fact that there exists a value of $p d$ corresponding to each value of $\sigma$ for which (5.13) is satisfied allows the following numerical procedure to find a lower bound for $K$ to be adopted. The function

$$
K_{1} a(\sigma)=p a \sin \sigma \tanh [p d(\sigma) \sin \sigma]
$$


is maximized over the range $\sigma \in\left[0, \frac{1}{2} \pi\right]$, where $p a$ is given and $p d(\sigma)$ satisfies

$$
t(p d)=0,
$$

where

$$
t=\min [s( \pm a g(y / a), y)], \quad 0 \leqslant y \leqslant h .
$$

This procedure will always generate a lower bound for $K$ and the accuracy of this bound for circular basins of arbitrary fill depth is tested in the next section. The maximization and minimization procedures and the finding of a zero of the function $t(p d)$ are performed numerically using NAG library routines.

Two trial functions for $w$ are used to obtain a lower bound for the value of $K$ associated with the lowest antisymmetric mode, namely

and

$$
\begin{gathered}
w_{1}=\sinh \left[\left(p^{2}-k^{2}\right)^{\frac{1}{2}}\left(x+\epsilon_{1} a\right)\right] \cosh k_{1}\left(y-d_{1}\right) \\
w_{2}=\sin \left[\left(k_{2}^{2}-p^{2}\right)^{\frac{1}{2}}\left(x+\epsilon_{2} a\right)\right] \cosh k_{2}\left(y-d_{2}\right)
\end{gathered}
$$

where $\epsilon_{1}, \epsilon_{2}>0, k_{1} d_{1}, k_{2} d_{2} \geqslant 0,0 \leqslant k_{1} a<p a$ and $0<\left(k_{2}^{2}-p^{2}\right)^{\frac{1}{2}}\left(x_{\mathrm{m}}+\epsilon_{2} a\right) \leqslant \frac{1}{2} \pi, x_{\mathrm{m}}$ being the maximum value $x$ takes in the container. The parameter $K_{1}$ associated with the $i$ th trial function, $K_{1 i}$, is defined by

$$
K_{1 i}=k_{i} \tanh k_{i} d_{i} \text {. }
$$

Clearly, both functions are positive in $x \geqslant 0$ and satisfy (5.1) and (5.3), putting $\beta=p$ and $K=K_{1 i}$. Condition (5.2) is satisfied by choosing $k_{1} a$ and $k_{1} d$ such that

$$
\left(p^{2}-k_{1}^{2}\right)^{\frac{1}{2}} a n_{x}+k_{1} a n_{y} \tanh \left[\left(p^{2}-k_{1}^{2}\right)^{\frac{1}{2}}\left(x+\epsilon_{1} a\right)\right] \tanh k_{1}\left(y-d_{1}\right) \geqslant 0 \text { for } x \geqslant 0 \text { on } C
$$

and choosing $k_{2} a$ and $k_{2} d$ such that

$$
\begin{aligned}
& \left(k_{2}^{2}-p^{2}\right)^{\frac{1}{2}} a n_{x} \cos \left[\left(k_{2}^{2}-p^{2}\right)^{\frac{1}{2}}\left(x+\epsilon_{2} a\right)\right] \\
& \quad+k_{2} a n_{y} \sin \left[\left(k_{2}^{2}-p^{2}\right)^{\frac{1}{2}}\left(x+\epsilon_{2} a\right)\right] \tanh k_{2}\left(y-d_{2}\right) \geqslant 0 \text { for } x \geqslant 0 \text { on } C .
\end{aligned}
$$

If the body shape function $g(y / a)$ is concave then (5.20) and (5.21) are automatically satisfied for any permitted value of $k_{1} a$ and $k_{2} a$ by choosing $k_{1} d_{1}=k_{1} y_{\mathrm{m}}$ and $k_{2} d_{2}=k_{2} y_{\mathrm{m}}$, where $y_{\mathrm{m}} / a$ is the value at which $g$ takes its maximum. Thus, there exists a value of $k_{i} d_{i}$ corresponding to each value of $k_{i} a, i=1,2$ for which (5.20) and (5.21) are satisfied and so analogous numerical procedures to that described in (5.14)-(5.16) may be devised to obtain values for $K_{11} a$ and $K_{12 a}$. The actual value of $K_{1} a$ is chosen to be the larger of these two values. The parameters $\epsilon_{1}$ and $\epsilon_{2}$ are required to be positive to ensure the positivity of $w_{1}$ and $w_{2}$, however they may be arbitrarily small. Numerically, therefore, it is convenient to put $\epsilon_{1}$ and $\epsilon_{2}$ equal to zero. The only time that this is likely to be inaccurate is when applying the conditions (5.20) and (5.21) at the point $x=0$ on $C$. To ensure that these conditions are properly satisfied at this point, $k_{1} d_{1}$ and $k_{2} d_{2}$ are chosen to be less than or equal to $k_{1} h$ and $k_{2} h$ respectively, where $h$ is the maximum depth of the container. Such a choice ensures that both of the terms on the left-hand side of the inequalities in (5.20) and (5.21) are non-negative at $x=0$ on $C$.

\section{Discussion and numerical results}

Some general features of the solution for sloshing in a horizontal cylinder have been considered here. In particular, certain aspects of the low- and high-frequency behaviour have been identified. The high-frequency limit of modes has been found to depend on the angle of intersection $\gamma$ of the container with the free surface. It has been shown that for $0<\gamma<\frac{1}{2} \pi$ there is a correspondence between the lower sloshing modes 

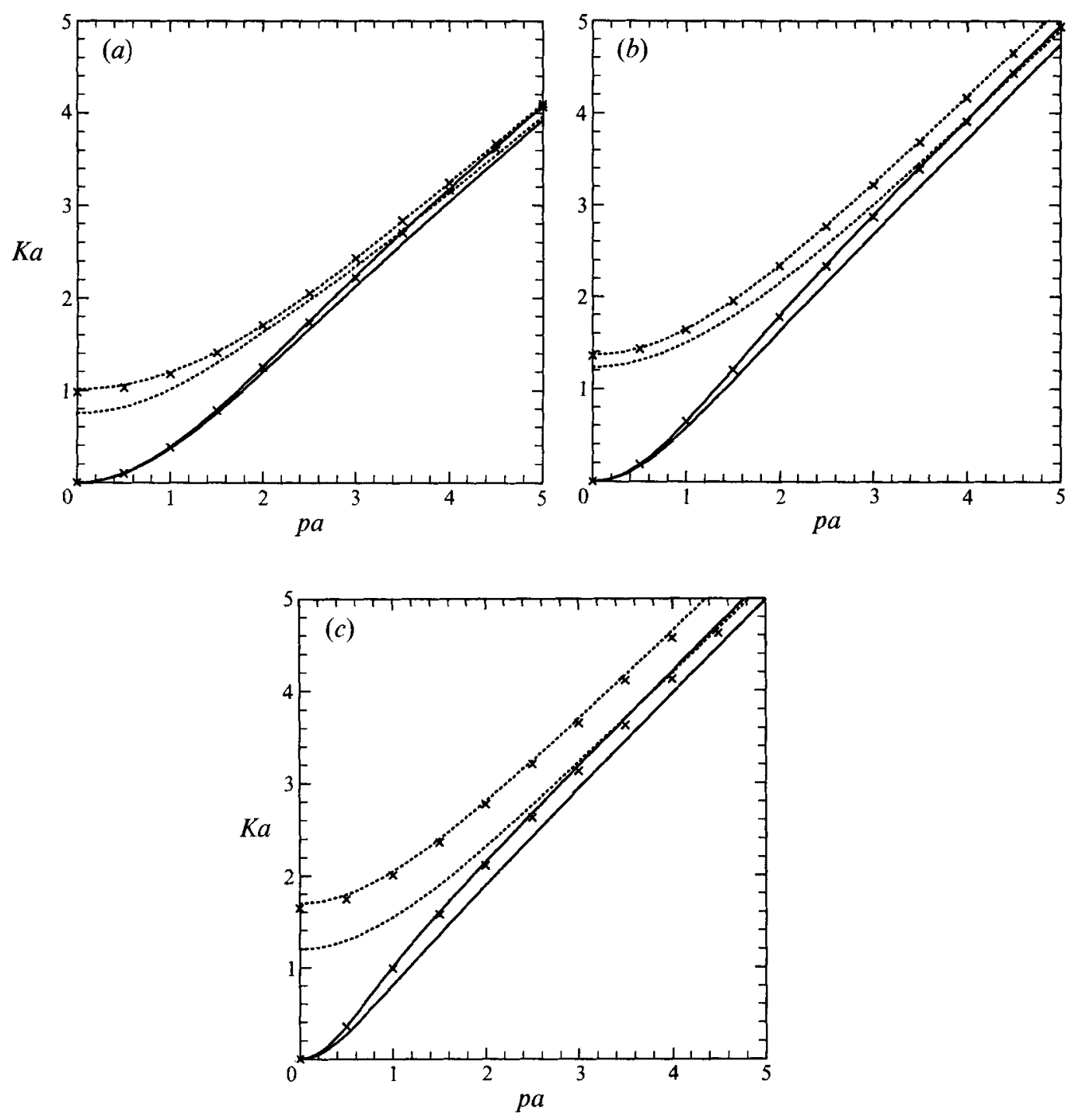

FIGURE 3. Comparison of the upper and lower bounds for the lowest symmetric mode (-) and the lowest antisymmetric mode (-- - ) with boundary element method calculations $(x)$ for a circular container of radius $c$ filled to depths $h$, where $(a) h / c=0.5,(b) h / c=1.0,(c) h / c=1.5$.

and edge-wave solutions for beaches of the same angle. In particular, for those modes the leading-order behaviour at high frequency depends on $\gamma$, as in equations (3.10). The leading-order behaviour for modes of a higher frequency than the edge-wave-type modes is independent of $\gamma$ and is given by (3.7). For containers where $\frac{1}{2} \pi \leqslant \gamma<\pi$, for example a container of circular cross-section more than or equal to half full, the highfrequency behaviour of all modes is governed by (3.7).

In $\$ 4$ and 5 methods have been given for calculating upper and lower bounds to the lowest symmetric and antisymmetric modes. Obtaining bounds for higher modes is a more complex task and is not pursued here. Numerical results for the present methods are presented in figure 3 for a container of circular cross-section filled to various depths and comparisons are made with calculations from a boundary-element method. The 
results are also typical of other geometries in that the upper bounds are generally closer to the exact solution than the lower bounds and the absolute errors for both bounds grow as $\gamma$ approaches $\pi$. Because of the restrictions on the trial function used to obtain the lower bound, it is not easy to generate other functions that would lead to improved lower bounds. The high-frequency behaviour is in accord with the results described above, in particular for $h / c=0.5$, where $h$ is the fill depth and $c$ the cylinder radius, the solutions asymptote to $K=p \sin \gamma$ where $\cos \gamma=1-h / c$. Calculations have been made by Evans \& Linton (1993) for the half-full case $h / c=1$ and the present boundary-element computations are in close agreement with these.

For a horizontal cylinder the most important modes of fluid oscillation are the transverse and longitudinal modes. Transverse oscillations correspond to $p=0$ in (1.6), so that the fluid motion is two-dimensional, and these have been studied extensively. A calculation method for these modes in a circular cylinder filled to arbitrary depth has recently been given by McIver (1989). For the cases given here in figure 3, the values of $K a$ predicted by McIver's method for the lowest transverse antisymmetric mode are $(a)$ 0.97687, (b) $1.35573,(c) 1.64448$. Longitudinal modes correspond to the lowest symmetric mode for non-zero $p$. From (1.5), a container of length $l$ can support longitudinal oscillations provided $p=m \pi / l$, where $m$ is a positive integer. The bounds given in the present paper work well for this important case. If the container is sufficiently long for $p$ to satisfy $p a \ll 1$ then the eigenfrequencies are given approximately in terms of the cross-sectional area of the container by (2.21).

\section{Appendix}

In this appendix, the theorem stated in $\S 5$ is proved. The proof follows that given for a more general theorem in Protter \& Weinberger (1984, p. 91).

Let $\phi$ be a non-zero solution of (1.6)-(1.8). Without loss of generality, $\phi$ is assumed to be real as the real and imaginary parts of any solution must separately satisfy each of the equations. The function $v$ is defined by

$$
v=\phi / w \text {. }
$$

Substitution of (A 1) into (1.6) yields

$$
\nabla^{2}(v w)-p^{2} v w=0
$$

After some manipulation this equation may be rewritten as

$$
\mathrm{L}[v]=-(v / w)\left[\nabla^{2} w-p^{2} w\right]
$$

where $L$ is the uniformly elliptic operator defined by $L=\nabla^{2}+(2 / w) \nabla w \cdot \nabla$. Applying the operator $\mathrm{L}$ to $v^{2}$ yields

$$
\begin{aligned}
\mathrm{L}\left[v^{2}\right] & =2(\nabla v)^{2}+2 v \nabla^{2} v+\frac{4 v}{w} \nabla w \cdot \nabla v \\
& =2(\nabla v)^{2}+2 v \mathrm{~L}[v], \\
& \geqslant-\frac{2 v^{2}}{w}\left[\nabla^{2} w-p^{2} w\right],
\end{aligned}
$$

using (A 3). Suppose that $p>\beta$, contradicting the result of the theorem, then

$$
\nabla^{2} w-p^{2} w<\nabla^{2} w-\beta^{2} w \leqslant 0
$$


using (5.1) and noting that $w$ is positive. Substitution of (A 5) into (A 4) gives

$$
\mathrm{L}\left[v^{2}\right] \geqslant 0 \text {. }
$$

The operator $\mathrm{L}$ is uniformly elliptic in $D$ and so $v^{2}$ satisfies a maximum principle. The implication of this principle (described by Protter \& Weinberger 1984) is that $v^{2}$ is either constant throughout $D$ or it attains a maximum on the boundary of $D$ and at such a point $\left(v^{2}\right)_{n}>0$, where ()$_{n}$ denotes the outward normal derivative to $D$. Differentiating (A 1) gives

$$
\left(v^{2}\right)_{n}=\left(2 v / w^{2}\right)\left[w \phi_{n}-\phi w_{n}\right] .
$$

The body boundary condition (1.7) requires $\phi_{n}=0$ on $C$, thus

$$
\left(v^{2}\right)_{n}=-\left(2 v^{2} / w\right) w_{n} \leqslant 0 \text { on } C,
$$

using (5.2). The free-surface condition (1.8) yields $\phi_{n}=K \phi$ on $F$ and so

$$
\left(v^{2}\right)_{n}=\left(2 v^{2} / w\right)\left[K w+w_{y}\right] \leqslant 0 \text { on } F,
$$

using (5.3). Thus, $\left(v^{2}\right)_{n} \leqslant 0$ everywhere on the boundary of $D$ and so $v^{2}$ must be constant throughout $D$. From (A 1 ) this means that

$$
\phi=A w,
$$

where $A$ is a constant. If $A$ is non-zero then substituting (A 10) into (1.6) gives

$$
\nabla^{2} w-p^{2} w=0
$$

which contradicts (A 5). Thus $A$ and therefore $\phi$ must equal zero which contradicts the original assumption that $\phi$ is a non-zero solution of (1.6)-(1.8). Thus, the supposition that $p>\beta$ leads to a contradiction and so

$$
p \leqslant \beta .
$$

\section{REFERENCES}

Abramowitz, M. \& Stegun, I. A. 1965 Handbook of Mathematical Functions. Dover.

Courant, R. \& Hilbert, D. 1953 Methods of Mathematical Physics, vol. 1. Interscience.

Davis, A. M. J. 1965 Two-dimensional oscillations in a canal of arbitrary cross-section. Proc. Camb. Phil. Soc. 61, 827-846.

DAvis, A. M. J. 1992 Discussion related to Watson and Evan: 'Resonant frequencies of a fluid in containers with internal bodies'. $J$. Engng Math. 26, 445-454.

Evans, D. V. \& Linton, C. M. 1993 Sloshing frequencies. Q. J. Mech. Appl. Maths (to appear).

Evans, D. V. \& MCIver, P. 1991 Trapped waves over symmetric thin bodies. J. Fluid Mech. 223, 509-519.

Fox, D. W. \& Kuttler, J. R. 1983 Sloshing frequencies. Z. Angew Math. Phys. 34, 668-696.

IsaAcson, E. 1950 Water waves over a sloping beach. Commun. Pure Appl. Math. 3, 1-32.

Kobayashi, N., Mieda, T., Shibata, H. \& Shinozaki, Y. 1989 A study of the liquid slosh response in horizontal cylindrical tanks. J. Pressure Vessel Technol. 111, 32-38.

Lamb, H. 1932 Hydrodynamics, 6th Edn. Cambridge University Press.

MCIVER, P. 1989 Sloshing frequencies for cylindrical and spherical containers filled to an arbitrary depth. J. Fluid Mech. 201, 243-257.

MCIver, P. \& SmIth, S. R. 1987 Free-surface oscillations of fluid in closed basins. J. Engng Math. 21, 139-148.

Moiseev, N. N. \& Petrov, A. A. 1966 The calculation of free oscillations of a liquid in a motionless container. Adv. Appl. Mech. 9, 91-154.

Paскнам, B. A. 1980 Small-amplitude waves in a straight channel of uniform triangular crosssection. Q. J. Mech. Appl. Math. 33, 179-187. 
Peters, A. S. 1952 Water waves over sloping beaches and the solution of a mixed boundary value problem for $\nabla^{2} \phi-k^{2} \phi=0$ in a sector. Commun. Pure Appl. Maths 5, 87-108.

Protter, M. H. \& Weinberger, H. F. 1984 Maximum Principles In Differential Equations. Springer.

SHI, J. \& YIH, C.-S. 1984 Waves in open channels. J. Engng Mech. 110, 847-870.

UrSell, F. 1952 Edge waves on a sloping beach. Proc. R. Soc. Lond. A 214, 79-97. 